\title{
ESTIMATIVAS DE PARÂMETROS GENÉTICOS PARA CARACTERÍSTICAS INCLUÍDAS EM CRITÉRIOS DE SELEÇÃO EM GADO NELORE ${ }^{1}$
}

\author{
ESTIMATES OF GENETIC PARAMETERS FOR THE TRAITS INCLUDED IN SELECTION \\ CRITERIA FOR NELORE CATTLE
}

\author{
Ricardo José Gunski ${ }^{2}$ Analía del Valle Garnero ${ }^{3}$ Arcadio de los Reyes Borjas ${ }^{4}$ \\ Luiz Antônio Framartino Bezerra ${ }^{5}$ Raysildo Barbosa Lôbo ${ }^{6}$
}

RESUMO

Foram analisados registros de 20 rebanhos integrantes do Programa de Melhoramento Genético da Raça Nelore (PMGRN), estudando-se as seguintes variáveis: ganho de peso por dia de idade até os 120,200,310 e 350kg (GPDpesok), peso padrão aos 120, 240, 365 e 550 dias de idade e peso real às idades mencionadas. As análises unicaráter mediante Modelo Animal, usando o aplicativo MTDFREML, proporcionaram estimativas de herdabilidades semelhantes para os pesos prédesmama, variando entre 0,21-0,27 para o efeito direto, sendo que a correlação genética entre efeitos diretos e maternos foi negativa, variando entre -0,42 a -0,24. Para os pesos pósdesmama os coeficientes de herdabilidade foram maiores para as variáveis pesos padrão e peso real do que em relação ao ganho de peso por dia de idade. A correlação genética mostrou a mesma tendência para os dois primeiros tipos de características e, para última, foi positiva e próxima de zero. A contribuição do efeito aditivo materno e do ambiente permanente para a variância fenotípica na maioria das características estudadas justificam sua inclusão em programas de Melhoramento genético na raça Nelore. Os resultados indicam que, para o período pós-desmama, a seleção por peso seria mais eficiente do que para o GPD.

Palavras-chave: nelore, modelo animal, parâmetros genéticos, critérios de seleção.

\section{SUMMARY}

Records of animals from 20 herds participating in the Genetic Breeding Program of Nelore Cattle (PMGRN) were analyzed. The variables considered were gains by day of age until
$120,200,310$ and $350 \mathrm{~kg}$ (WGD weight $\mathrm{k}$ ), the adjusted weights at 120, 240, 365 and 550 days and the observed weights at these same ages. Animal Model analysis using MTDFREML programs showed similar heritabilities for pre-weaning weights ranging from 0.21 to 0.27 for direct effect. The genetic correlation between direct and maternal effects was negative and ranged from -0.42 to -0.24 . For postweaning weights the heritabilities were greater for the adjusted and observed weights then for the daily gain by age. The genetic correlation showed the same tendency for the first two kinds of traits and was positive and close to zero for the last one. The contribution of additive maternal effect and permanent environmental effect to the phenotypic variance of most traits studied should be considered in breeding programs of Nelore cattle. Results suggested that selection for postweaning traits would be more efficient when done on weights than on WGD.

Key words: nelore, animal model, genetic parameters, selection criteria.

\section{INTRODUÇÃO}

A pecuária de corte no Brasil atravessa fase de crescimento e de grande interesse na aplicação de tecnologias. A adoção dessas novas tecnologias é de fundamental importância para que possa haver ganho em eficiência e qualidade, permitindo maior competição frente à unificação dos países em vários blocos econômicos (LÔBO et al., 1998).

\footnotetext{
${ }^{1}$ Apoio Financeiro: FINEP, RHAE, FUNPEC, CAPES. Autor para correspondência.

${ }^{3}$ Licenciatura em Genética, Mestre, Doutoranda da FMRP, USP.

${ }^{4}$ Zootecnista, Doutor, Professor Titular, Universidade Federal de Goiânia.

${ }^{5}$ Analista de Sistemas, Mestre, Departamento de Genética, FMRP, USP.

${ }^{6}$ Médico Veterinário, Doutor, Professor Adjunto, Departamento de Genética, FMRP, USP.

${ }^{2}$ Licenciatura em Genética, Mestre, Professor Adjunto, Departamento de Genética, FCEQyN. UNaM. Argentina. Doutorando da FMRP, Universidade de São Paulo (USP). Av. Bandeirantes 3900, 14049-900, Ribeirão Preto, SP. Brasil. E-mail: rgunski@rgm.fmrp.usp.br. 
A grande capacidade de adaptação ao clima tropical, no qual se destacam a resistência à ecto- e endoparasitos, altas temperaturas e pobres condições de manejo e alimentação, fazem do zebu o biótipo ideal para a exploração agropecuária, constituindo a raça Nelore aproximadamente $60 \%$ do rebanho nacional brasileiro estimado em 146,1 milhões de cabeças (FNP, 1998). Seu papel no aumento da produtividade dos rebanhos como raça pura ou em cruzamentos é de grande importância, considerando-se a alta rusticidade e fertilidade que apresenta (ELER et al., 1996).

As estimativas de parâmetros genéticos para características ponderais na raça Nelore têm apresentado grande variabilidade, sendo um bom indicador da resposta à seleção ou melhoramento genético a ser alcançado. Estes parâmetros são necessários para estimar as respostas diretas e correlacionadas à seleção, para elaborar índices de seleção e predizer o valor genético dos animais.

Os pesos e ganhos em peso a diferentes idades têm sido frequientemente incluídos nos programas de seleção em gado de corte, baseados fundamentalmente nas altas correlações existentes entre eles. CATER (1971) analisou o efeito da eficiência da seleção de touros com base na correlação do peso à desmama com o peso final e concluiu que a seleção, baseada no peso à desmama ou ao ano, pode resultar em bom ganho genético. Utilizando dados de bezerros Hereford, PONS et al. (1990) determinaram índices de seleção incluindo as características de peso e escore de conformação do indivíduo ao desmame, ao ano e ao sobreano. Com base na estimativa de correlação $(0,98 \pm 0,03)$, os autores concluíram que deve existir melhoria de conformação pela seleção direta para peso ao desmame. No gado Canchim MASCIOLI et al. (1996) concluíram que pesos aos 12 e 18 meses de idade são bons critérios de seleção para aumentar o peso.

ELER et al. (1995a) estimaram, na raça Nelore, componentes de variância e parâmetros genéticos para peso aos 205 (P205) e 365 (P365) dias de idade por Modelo Animal, obtendo valores de herdabilidade para efeito genético direto $\left(\mathrm{h}^{2}{ }_{\mathrm{a}}\right)$ de 0,13 (P205) e 0,16 (P365), o efeito aditivo materno $\left(\mathrm{h}^{2}{ }_{\mathrm{m}}\right)$ foi de 0,13 (P205) e 0,10 (P365). Em estudos posteriores, ELER et al. (1995b, 1996), além das características acima mencionadas, incorporaram o peso ao sobreano (P550), estimando valores de $h^{2}{ }_{a}$ $=0,30$ e 0,29 , respectivamente. No entanto, estimativas superiores para P205 $\left(\mathrm{h}_{\mathrm{a}}^{2}=0,58\right)$ e P365 $\left(\mathrm{h}_{\mathrm{a}}^{2}\right.$ $=0,69$ ) foram obtidos por MAGNABOSCO $\boldsymbol{e t}$ al . (1995) analisando dados de um único rebanho.

No Brasil escassos estudos incluindo ganhos em peso como critério de seleção têm sido realizados, observando-se estimativas de herdabilidades para ganhos pré-desmama entre 0,17 a 0,25 e entre 0,24 a 0,57 para pós-desmama (MERCADANTE $\boldsymbol{e t}$ al., 1995; PAZ, et al., 1997; REYES et al., 1997; ORTIZ PEÑA, 1998).

O objetivo do presente estudo foi estimar componentes de (co)variância genéticas e de ambiente para as variáveis ganho em peso por dia de idade (GPD) até o peso considerado $(120,200,310$, $350 \mathrm{~kg}$ ) e os pesos padronizados (p) e reais (r) (P120, P240, P365, P550) como critérios de seleção na raça Nelore.

\section{MATERIAL E MÉTODOS}

Analisaram-se 24.661 registros de 20 rebanhos integrantes do Programa de Melhoramento Genético da Raça Nelore (PMGRN), no período 1985-1995, estudando-se as seguintes variáveis: ganho em peso por dia de idade até os 120, 200, 310 e $350 \mathrm{~kg}$ (GPD peso $\mathrm{k}$ ), correspondentes em média às idades de 4, 8, 18 e 20 meses; peso padrão para 120, 240,365 e 550 dias de idade e peso real às idades mencionadas.

Os cálculos foram feitos como segue: GPD peso $\mathrm{k}=$ peso/IDA peso $\mathrm{k}$; Peso padrão = $\mathrm{p}_{\mathrm{a}}+\mathrm{GMD} \times \mathrm{d}_{\mathrm{a}}$; Peso real = peso observado; onde: GPD peso $\mathrm{k}=$ ganho em peso por dia de idade até o peso considerado (ex. 120); IDA peso $\mathrm{k}=$ idade do animal ao atingir o "peso"; Peso padrão = peso às idades $120,240,365$ e 550 dias; $\mathrm{p}_{\mathrm{a}}=$ peso anterior à idade padrão; $\mathrm{d}_{\mathrm{a}}=$ dias compreendidos entre a pesagem anterior e a idade padrão; GMD (Ganho Médio Diário) $=$ razão da diferença entre os pesos anteriores e posteriores pelo número de dias entre as duas pesagens.

Para a obtenção de estimativas das (co)variâncias e parâmetros genéticos foi utilizado o software MTDFREML (BOLDMAN et al., 1995).

As características analisadas foram descritas segundo o seguinte modelo misto:

$\mathbf{Y}=\mathbf{X} \beta+\mathbf{Z}_{1} \mathbf{u}+\mathbf{Z}_{2} \mathbf{m}+\mathbf{Z}_{\mathbf{3}} \mathbf{p}+\mathbf{e}$

onde: $\mathrm{Y}=$ vetor das observações de cada característica; $\mathrm{X}=$ matriz de incidência dos efeitos fixos; $\beta=$ vetor dos efeitos fixos, incluindo a subclasse rebanho-ano-trimestre-sexo-manejo e classe de idade da vaca ao parto; $Z_{1}=$ matriz de incidência do efeito genético direto de cada animal; $\mathrm{u}=$ vetor de efeitos genéticos diretos aleatórios; $Z_{2}=$ matriz de incidência dos efeitos genéticos maternos; $\mathrm{m}=$ vetor de efeitos genéticos maternos aleatórios; $Z_{3}=$ matriz de incidência do efeito do ambiente permanente; $\mathrm{p}=$ vetor de efeitos aleatórios do ambiente permanente; $\mathrm{e}=$ vetor de efeitos residuais aleatórios. 
Para a característica peso real, a idade do animal por ocasião da pesagem foi incluída como covariável no modelo.

As pressuposições acerca da distribuição de $y, a, m, p$ e $\varepsilon$ são descritas como:

$$
\operatorname{Var}\left(\begin{array}{l}
u \\
m \\
\mathrm{p} \\
\varepsilon
\end{array}\right)=\left(\begin{array}{llll}
A \sigma_{u}^{2} & A \sigma_{u m} & 0 & 0 \\
A \sigma_{u m} & A \sigma_{m}^{2} & 0 & 0 \\
0 & 0 & I_{c} \sigma_{p}^{2} & 0 \\
0 & 0 & 0 & I_{N} \sigma_{e}^{2}
\end{array}\right)
$$

\section{RESULTADOS E DISCUSSÃO}

No tabela 1, são apresentados os componentes de (co)variância obtidos nas análises unicaráter, estimativas das variâncias genéticas, de ambiente permanente, residuais, herdabilidades e correlações genéticas entre efeitos diretos e maternos. Nas características estudadas, os valores de herdabilidade observados são semelhantes para os pesos prédesmama, variando entre $0,21-0,27$ para $^{2}{ }_{a}$ e entre $0,08-0,16$ para $\mathrm{h}_{\mathrm{m}}^{2}$, sendo que a correlação genética entre efeito direto e materno foi negativa e variou entre $-0,42$ a $-0,24$.

Para os pesos pós-desmama, os coeficientes de herdabilidade obtidos foram sensivelmente maiores para as características pesos padrão e peso real $(0,30$ a 0,37$)$ em relação ao ganho de peso por

Tabela 1 - Componentes de (co)variâncias e parâmetros genéticos obtidos das análises unicarácter para as características estudadas em bovinos da raça Nelore.

\begin{tabular}{|c|c|c|c|c|c|c|c|c|c|}
\hline Característica $^{\mathrm{b}}$ & $\sigma_{\mathrm{a}}^{2}$ & $\sigma_{\mathrm{m}}^{2}$ & $\sigma_{\mathrm{am}}$ & $\sigma_{\mathrm{pe}}$ & $\sigma_{\mathrm{e}}$ & $\mathrm{h}_{\mathrm{a}}^{2}$ & $\mathrm{~h}_{\mathrm{m}}^{2}$ & $\mathrm{r}_{\mathrm{am}}$ & $c^{2}$ \\
\hline GPD120k & 6319,0 & 4357,0 & $-1829,0$ & 3033,0 & 17987 & 0,21 & 0,15 & $-0,35$ & 0,10 \\
\hline GPD200k & 5725,0 & 1883,0 & $-1183,0$ & 1405,0 & 14999 & 0,25 & 0,08 & $-0,36$ & 0,06 \\
\hline GPD310k & 1219,0 & 315,0 & 37,0 & 0,4 & 4578 & 0,20 & 0,05 & 0,06 & 0,00 \\
\hline GPD350k & 1096,0 & 141,0 & 31,0 & 197,0 & 4754 & 0,18 & 0,02 & 0,08 & 0,03 \\
\hline P120p & 59,0 & 34,0 & $-19,0$ & 24,0 & 120 & 0,27 & 0,16 & $-0,42$ & 0,11 \\
\hline P120r & 64,0 & 35,0 & $-17,5$ & 26,0 & 157 & 0,26 & 0,10 & $-0,24$ & 0,09 \\
\hline P240p & 126,0 & 49,0 & $-19,0$ & 45,5 & 275 & 0,26 & 0,10 & $-0,24$ & 0,09 \\
\hline $\mathrm{P} 240 \mathrm{r}$ & 131,5 & 51,0 & $-20,0$ & 45,0 & 344,6 & 0,24 & 0,09 & $-0,24$ & 0,08 \\
\hline P365p & 186,0 & 61,0 & $-41,0$ & 38,0 & 384 & 0,30 & 0,10 & $-0,38$ & 0,06 \\
\hline $\mathrm{P} 365 \mathrm{r}$ & 240,0 & 66,5 & $-67,6$ & 53,6 & 474,3 & 0,31 & 0,09 & $-0,53$ & 0,06 \\
\hline P550p & 334,0 & 372,0 & 1,0 & 20,6 & 563,1 & 0,35 & 0,04 & 0,01 & 0,02 \\
\hline P550r & 416,5 & 30,2 & $-32,0$ & 43,5 & 660,5 & 0,37 & 0,03 & $-0,29$ & 0,03 \\
\hline
\end{tabular}

$\sigma^{2}{ }_{a}=$ variância genética aditiva, $\sigma_{m}^{2}=$ variância genética maternal, $\sigma_{a m}=$ covariância genética entre efeitos diretos e maternal, $\sigma_{\mathrm{pe}}^{2}$ variância do ambiente permanente, $\sigma_{\mathrm{e}}^{2}=$ variância residual, $\mathrm{h}_{\mathrm{a}}^{2}=$ herdabilidade direta, $\mathrm{h}_{\mathrm{m}}^{2}=$ herdabilidade maternal, $\mathrm{r}_{\mathrm{am}}=$ correlação genética entre efeitos genético e maternal, $\mathrm{c}^{2}=$ proporção de variância devida ao efeito permanente do ambiente.

${ }^{\mathrm{b}}$ GPDpesok = ganho de peso por dia de idade até o peso considerado (120 a 350), Pdiasp = peso padrão em dias (120 a 550), Pdiasr = peso real em dias (120 a 550). dia de idade $(0,18$ a 0,20$)$, a herdabilidade para efeito materno foi próxima de 0,10 para P365r e P365p e sensivelmente menor para GPD350k $(0,02)$. A correlação genética mostrou a mesma tendência nas duas primeiras características e foi positiva e próxima de zero na última.

Os valores obtidos no presente trabalho foram consistentemente maiores que os relatados por ELER (1995a), porém inferiores aos citados por MAGNABOSCO et al. (1995), embora nesse trabalho tenham sido considerados dados de um único rebanho e os autores apontem para a estrutura dos ados e a inadequada distribuição dos touros dentro e entre anos.

A contribuição do ambiente permanente $\left(c^{2}\right)$ para a variância fenotípica foi importante $(0,10)$ para todas as características analisadas na fase prédesmama, sendo desprezível sua contribuição na fase pós-desmama $(0,02)$, resultados semelhantes os obtidos por ELER et al. (1996). Esses autores, baseados nas altas correlações do peso à desmama e sobreano $(0,77)$ sugerem o primeiro como um bom critério de seleção apesar da influência dos efeitos maternos.

Recentemente vários autores têm proposto critérios de seleção alternativos para características de crescimento, como dias, para atingir $160 \mathrm{~kg}$ entre nascimento e à desmama (D160) e dias para atingir $240 \mathrm{~kg}$ entre a desmama e o ponto final (D240) (FRIES \& ALBUQUERQUE, 1996) e estimaram coeficientes de herdabilidades para efeito genético aditivo direto $(0,40)$ e efeito aditivo materno $(0,12)$ para a característica D160, sugerindo que sua utilização como objetivo de seleção, em relação ao ganho do nascimento à desmama (GND), permitiria obter um progresso genético 54\% superior. MARCONDES et al. (1998), ORTIZ PEÑA, (1998) e GARNERO (1999), porém, encontraram valores de $\mathrm{h}_{\mathrm{d}}^{2}=0,14 \mathrm{e} \mathrm{h}_{\mathrm{m}}{ }_{\mathrm{m}}=0,05$ 0,09 para a característica D160, utilizando diferentes conjuntos de dados. Esses resultados, sensivelmente inferiores aos citados anteriormente, indicam a necessidade de maiores estudos sobre tal parâmetro para a característica considerada.

Nesse mesmo sentido, REYES et al. (1997) analisaram várias características como: ganho de peso por dia de idade, idades reais as quais esses pesos são alcançados e ganho médio diário entre outras. Concluíram que as idades às quais os pesos são 
alcançados após à desmama seriam mais vantajosas para a seleção que os próprios pesos. No entanto, LÔBO et al. (1997b) comparando os parâmetros genéticos das características alternativas estudadas por REYES et al. (1997) com aqueles dos pesos padronizados utilizados no Programa de Melhoramento Genético da Raça Nelore (PMGRN), consideraram a estes últimos mais adequados que outros critérios de seleção alternativos. O PMGRN tem promovido no período 1988-1998 uma redução no número de dias para os animais atingirem $350 \mathrm{~kg}$ nos machos e $300 \mathrm{~kg}$ nas fêmeas respectivamente, assim como mudanças genéticas relevantes para a maioria das características analisadas (LÔBO et al., 1997a; 1997b; 1998).

ROSA (1999) analisou 6907 registros de pesos no animal adulto (considerando o primeiro peso após os 4 anos de idade) de matrizes na Raça Nelore, no período 1982-1997 e verificou um aumento do peso adulto de 0,416kg/ano. Essa observação vem reforçar os conceitos sobre a vantagem da utilização dos pesos como critérios de seleção para características de crescimento, em contraposição à idéia de que a seleção para pesos conduz a aumento no peso adulto.

\section{CONCLUSÃO}

Os valores obtidos para herdabilidade materna e correlação genética entre efeitos diretos e maternos apontam para a inclusão desse último nos programas de seleção e avaliação genética da raça Nelore, assim como o efeito do ambiente permanente na fase pré-desmama.

Embora seja reduzido o número de trabalhos em zebuínos com estimativas de parâmetros genéticos do ganho de peso por dia de idade, os resultados deste estudo indicam que a seleção sobre os pesos seria mais eficiente que sobre os ganhos de peso no período pós-desmama.

\section{AGRADECIMENTOS}

Os autores agradecem aos Criadores do Programa de Melhoramento Genético da Raça Nelore.

\section{REFERÊNCIAS BIBLIOGRÁFICAS}

BOLDMAN, K.G., KRIESE, L.A., VAN VLECK, L.D., et $\boldsymbol{a l}$. A manual for use for MTDFREML. A set of programs to obtain of variance and covariances [DRAFT]. Lincoln : Agricultural Reserach Service, 1995. 120p.
CATER, A.H. Effectiveness of growth performance in cattle. Proceedings of New Zeland Society of Animal Production, v.31.p.151-163.1971.

ELER, J.P., VAN VLECK, L.D., FERRAZ, J.B.S., et al. Estimation of variances due to direct and maternal effects for growth traits of Nelore cattle. J Anim Sci, v.77, p.3253-3258, 1995a.

ELER, J.P., FERRAZ, J.B.S, SILVA, P.R. Parâmetros genéticos de pesos de bovinos da raça Nelore. In: REUNIÃO ANUAL DA SOCIEDADE BRASILEIRA DE ZOOTECNIA, 32, 1995b, Brasilia, DF. Anais... Brasilia : Sociedade Brasileira de Zootecnia, 1995. p.709-710.

ELER, J.P., FERRAZ, J.B.S., SILVA, P.R. Estimação simultânea de parâmetros de importância econômica na raça Nelore, com a utilização de modelos animais. I n: REUNIÃO ANUAL DA SOCIEDADE BRASILEIRA DE ZOOTECNIA, 33, 1996, Fortaleza, CE. Anais... Fortaleza : Sociedade Brasileira de Zootecnia, 1996. p.99-101.

FNP CONSULTORIA \& COMERCIO. Anuário da pecuária Brasileira. São Paulo, Brasil : ANUALPEC 98, 1998. 385p.

FRIES, L.A., ALBUQUERQUE, L.G. Avaliação genética de duas alternativas para medir precocidade de crescimento. In: SIMPÓSIO NACIONAL DE MELHORAMENTO ANIMAL, 1, 1996, Ribeirão Preto, SP. Anais... Ribeirão Preto : Sociedade Brasileira de Melhoramento Animal, 1996. p.243-245.

GARNERO, A. del V. Comparação de critérios de seleção em gado de corte visando precocidade de crescimento. Ribeirão Preto, SP, 1999. 85p. Dissertação (Mestrado em Genética) - Curso de Pós-graduação em Ciências, FMRP, Universidade de São Paulo, 1999.

LÔBO, R.B., REYES, A. de los, BEZERRA, L.A.F. Avaliação genética de animais jovens, touros e matrizes - Sumário 1997. Ribeirão Preto, SP : Departamento de Genética FMRP - USP, 1997a. 65p.

LÔBO, R.B., REYES, A. de los, BITTENCOURT, T.C.C., et al. Programa de melhoramento genético da raça Nelore: Resultados. In: SIMPÓSIO: O NELORE DO SÉCULO XXI, 4, 1997, Uberaba, MG. Anais... Uberaba : Associação Brasileira dos Criadores de Zebu 1997b. p.147-162.

LÔBO, R.B., BEZERRA, L.A.F., OLIVEIRA, H.N., et al. Avaliação genética de animais jovens, touros e matrizes - Sumário 1998. Ribeirão Preto, SP : Departamento de Genética - FMRP - USP, 1998. 60p.

MAGNABOSCO, C. DE U., FAMULA, T.R., LÔBO, R.B., et al. Componentes da variância e covariância para características de crescimento em um rebanho da raça Nelore mocho do Estado de São Paulo. In: REUNIÃO ANUAL DA SOCIEDADE BRASILEIRA DE ZOOTECNIA, 32, 1995, Brasília, DF. Anais... Brasília : Sociedade Brasileira de Zootecnia, 1995. p.677-679.

MASCIOLI, A.S., ALENCAR, M.M., BARBOSA, P.F. et al. Estimativas de parâmetros genéticos e proposição de critérios de seleção para pesos na raça Canchim. Revista da Sociedade Brasileira de Zootecnia, Viçosa v.25. n.1: p.72-82, 1996.

MARCONDES, R.C., BERGMANN, J.A.G., ELER, J.P., et al. Estimativas de componentes de variância e parâmetros gené 
ticos das características dias para $160 \mathrm{~kg}$ e taxas de crescimento relativo pré e pós-desmama, em animais da raça Nelore. In: CONGRESSO BRASILEIRO DAS RACAS ZEBUÍNAS, 3, 1998, Uberaba MG. Anais... Uberaba : Associação Brasileira dos Criadores de Zebu, 1998. p.385-387.

MERCADANTE, M.E.Z., LÔBO, R.B., REYES, A. de los B. Parámetros genéticos para características de crecimiento en cebuínos de carne. Arch Latinoam Prod Anim, v.3 n.1, p.45-89, 1995.

ORTIZ PEÑA, C.D. Análise de critérios de seleção para precocidade sexual e de crescimento de bovinos da raça Nelore, no Paraguai. Jaboticabal, SP, 1998. 104 p. Dissertação (Mestrado em Melhoramento Genético Animal) - Curso de Pós-graduação em Zootecnia, FCAVJ, Universidade Estadual Paulista, 1998.

PAZ, C.C.P. de, ALBUQUERQUE, L.G., FRIES, L.A. Contribuição ao estudo do coeficiente de repetibilidade do ganho de peso médio diário no período do nascimento ao desmame em bovinos da raça Nelore. In: REUNIÃO ANUAL DA
SOCIEDADE BRASILEIRA DE ZOOTECNIA, 34, 1997 Juiz de Fora MG. Anais... Juiz de Fora : Sociedade Brasileira de Zootecnia, 1997. p.152-154.

PONS, S.B, MILAGRES, J.C., REGAZZI, A.J. Efeitos de fatores genéticos e de ambiente sobre o crescimento e o escore de conformação ao sobreano. Revista da Sociedade Brasileira de Zootecnia, v.19 n.2, p.77-82, 1990.

REYES, A. de los B., LÔBO, R.B., BEZERRA, L.A.F., $\boldsymbol{e}$ t al. Variabilidade genética de características do crescimento alternativas para a seleção em gado de corte. In: REUNIÃO ANUAL DA SOCIEDADE BRASILEIRA DE ZOOTECNIA, 34, 1997 Juiz de Fora MG. Anais... Juiz de Fora : Sociedade Brasileira de Zootecnia, 1997. p.245-247.

ROSA, A.N. Variabilidade fenotípica e genética do peso adulto e da produtividade acumulada de matrizes em rebanhos de seleção da raça Nelore no Brasil. Ribeirão Preto, SP, 1999. 113p. Tese (Doutorado em Genética) - Curso de Pós-graduação em Ciências, FMRP Universidade de São Paulo, 1999.

Ciência Rural, v. 31, n. 4, 2001. 